\title{
Dietary vitamin B intake and the risk of esophageal cancer: a meta-analysis
}

This article was published in the following Dove Press journal: Cancer Management and Research

\author{
Jun-Li Ma' \\ Yan Zhao' \\ Chen-Yang Guo' \\ Hong-Tao $\mathrm{Hu}^{\prime}$ \\ Lin Zheng' \\ Er-Jiang Zhao ${ }^{2}$ \\ Hai-Liang Li' \\ 'Department of Radiology \\ Intervention, Affiliated Tumor Hospital \\ of Zhengzhou University, Zhengzhou \\ 450003, China; ${ }^{2}$ Department of \\ Epidemiology and Biostatistics, \\ Affiliated Tumor Hospital of \\ Zhengzhou University, Zhengzhou \\ 450003, China
}

Correspondence: Hai-Liang Li Department of Radiology Intervention, Affiliated Tumor Hospital of Zhengzhou University, 127 Dongming Road, Zhengzhou 450003, China Email hlli2016@I63.com
Background: Several epidemiology studies have explored the association between dietary B vitamins' intake and the risk of esophageal cancer (EC). However, the results remain inconclusive. Thus, we conducted a systematic review with meta-analysis to evaluate such association. Methods: Literature retrieval was performed using PubMed (Medline), ScienceDirect, and Cochrane Library electronic databases for all studies published from database inception to December 2017.

Results: The meta-analysis included 19 studies and showed an overall decreased risk of EC $(\mathrm{OR}=0.77,95 \%$ CI: 0.68-0.87) in association with multivitamin B (ie, B1, B2, B3, B5, B6, B9, and B12) dietary intake. In a subgroup analysis based on vitamin B subclass, B1, B3, B6, and $\mathrm{B} 9$ vitamins were associated with decreased EC risk (vitamin $\mathrm{B} 1$ : $\mathrm{OR}=0.68,95 \% \mathrm{CI}: 0.56-0.82$; vitamin B3: OR=0.70, 95\% CI: 0.53-0.94; vitamin B6: OR=0.64, 95\% CI: 0.49-0.83; and vitamin B9: $\mathrm{OR}=0.69,95 \% \mathrm{CI}: 0.55-0.86$ ). By contrast, no association was detected between dietary vitamin $\mathrm{B} 2$ and vitamin $\mathrm{B} 5$ intake and $\mathrm{EC}$ risk (vitamin $\mathrm{B} 2$ : $\mathrm{OR}=0.86,95 \% \mathrm{CI}$ : 0.64-1.16; vitamin $\mathrm{B} 5$ : $\mathrm{OR}=0.49,95 \% \mathrm{CI}: 0.20-1.20$ ), whereas a potential non-linear dose-response association was found between dietary vitamin B12 intake and EC risk. A statistically significant, inverse association was observed for an increase of $100 \mu \mathrm{g}$ /day in supplemental vitamin B6 and B9 and EC risk (vitamin B6: OR=0.98, 95\% CI: 0.98-0.99; vitamin B9: OR=0.89; 95\% CI: 0.86-0.94). Conclusion: These findings support that vitamin $\mathrm{B}$ may have an influence on carcinogenesis of the esophagus. Vitamin B1, B3, B6, B9 showed a decreased risk of EC, and vitamin B12 showed an increased risk of EC.

Keywords: B vitamins, esophageal cancer, meta-analysis

\section{Introduction}

Esophageal cancer (EC) has been ranked as the eighth most common cancer and the sixth leading cause of cancer-related deaths worldwide. ${ }^{1}$ Its epidemiology varies widely, particularly in incidence rates among geographic regions. ${ }^{2}$ The latest epidemiological studies indicated the highest rate of EC located on the "esophageal cancer belt" ie, China, South Africa, and France. ${ }^{3,4}$ Possible risk factors for EC include alcohol drinking, hot-temperature food items, cigarette smoking, chronic mucosal irritation, and a family history of cancers. ${ }^{5-7}$ Deficiency of nutrients, such as vitamins and microelements, was also found to be associated with an increased risk of EC, whereas a high intake of fruit and vegetables has been considered to be effective in prevention. ${ }^{6}$ Several previous research studies have evaluated the effect of beta-carotene, vitamin $\mathrm{A}, \mathrm{C}$, and $\mathrm{E}$ on $\mathrm{EC} .^{8-17}$ Regarding multivitamin $\mathrm{B}$, most studies only examined folate 
intake and EC risk, and no relevant pooled analyses have been performed. Thus, we conducted a meta-analysis of the current epidemiological articles to better characterize the association between multivitamin B intake and EC risk.

\section{Materials and methods}

\section{Search strategy}

We conducted a systematic search for published articles and abstracts that evaluated the relationships between $B$ vitamins (B1, B2, B3, B5, B6, B9, B12) and the risk of esophageal carcinoma in humans.

We conducted systemic searches of PubMed (Medline), ScienceDirect, and Cochrane Library electronic databases (from database inception to December 2017). The searches were performed using ((()cohort studies) OR case-control studies $))$ AND ( (()(()((()((vitamin B) OR vitamin B1) OR vitamin B2) OR vitamin B3) OR vitamin B5) OR vitamin B6) OR vitamin B9) OR vitamin B12) OR thiamin) OR riboflavin) OR pyridoxal) OR folate) OR cyanocobalamin)) AND (((((cancer) OR neoplasm) OR carcinoma)) AND Esophag*) in all fields. In addition, we scrutinized references from relevant original reports, review articles, and meta-analyses to identify other appropriate studies.

\section{Inclusion criteria}

In order to be included, the following criteria were needed: 1) the study was designed as a cohort, nested case-control or case-control study; 2) the study reported vitamin B and any kind of $\mathrm{B}$ vitamin group intake and the risk of $\mathrm{EC} ; 3$ ) the results reported effect estimates (RR, OR) and 95\% CIs for comparisons between high and low dietary vitamin $\mathrm{B}$ intake. When multiple levels of vitamin B intake were presented, the ratio comparing the highest intake vs the lowest intake was chosen. When data from several publications were overlapping, we selected the articles with the most comprehensive data for inclusion in this meta-analysis.

\section{Data extraction and quality assessment}

Two researchers independently reviewed titles and abstracts of potentially eligible research identified by the search strategy and extracted the date using a standard extraction form from each included publication: the first author's name, publication year, source of control, study design, country where the study was performed, type of cancer, specific vitamin measured, number of cases, number of controls or cohort size, total sample size, lowest vitamin B level, highest vitamin B level, difference between the highest and lowest vitamin B levels, and the risk estimates on EC and corresponding 95\%
CIs for the highest vs lowest categories of vitamin B intake or for each category, factors adjusted for. Adjusted ratios were extracted in preference to non-adjusted ratios.

Two authors independently assessed the quality of included studies using the Newcastle-Ottawa Scale (NOS), which is a validated scale for assessing the quality of nonrandomized studies in meta-analyses. ${ }^{18,19}$ This scale awards a maximum of 9 points to each study: 4 for selection of participants and measurement of exposure, 2 for comparability of cohorts on the basis of the design or analysis, and 3 for evaluation of methodological quality outcomes. We assigned scores of 7 or higher to high-quality studies. ${ }^{20,21}$

\section{Statistical analyses}

In this meta-analysis, we calculated effect estimates (RR or OR) and $95 \%$ CIs in each study to evaluate the relationship between vitamin $B$ intake and the risk of EC. We used a fixed effects model (Mantel-Haenszel method) when heterogeneity was negligible, and a random effects model (DerSimonian and Laird method) when heterogeneity was significant. Heterogeneity was assessed using $I^{2}$ statistic. Significant heterogeneity was indicated if $I^{2}$ values were greater than $50 \%{ }^{22,23}$ We also performed a sensitivity analysis by removing individual studies from the meta-analysis when statistically significant heterogeneity was detected. We also used Egger's and Begg's tests to assess publication bias. ${ }^{24,25}$ All tests were two-sided and results were regarded as statistically significant if $P<0.05$. All statistical analyses were done by using STATA software (version 12.0; StataCorp LP, College Station, TX, USA).

\section{Results}

\section{Literature search}

Figure 1 shows the literature search results and screening of this study. We identified 390 observational studies from PubMed (Medline), ScienceDirect, and Cochrane Library. A total of 332 articles were assessed after eliminating 58 duplicate papers. A total of 268 articles were excluded owing to reported irrelevant results after reviewing the title and abstract. In addition, three additional studies were found by a manual search of the reference lists. In total, full text of 67 articles was reviewed. Among them, 13 studies did not show the association of vitamin B and EC risk, because these 13 articles explored the relationship between nutrient intervention or mineral compound vitamin B or all the nutrient intake and risk of EC or precancerous lesions. Four articles did not report sufficient data for estimation of OR/RR, three articles did not separately report the $95 \% \mathrm{CI}$, nine articles were 


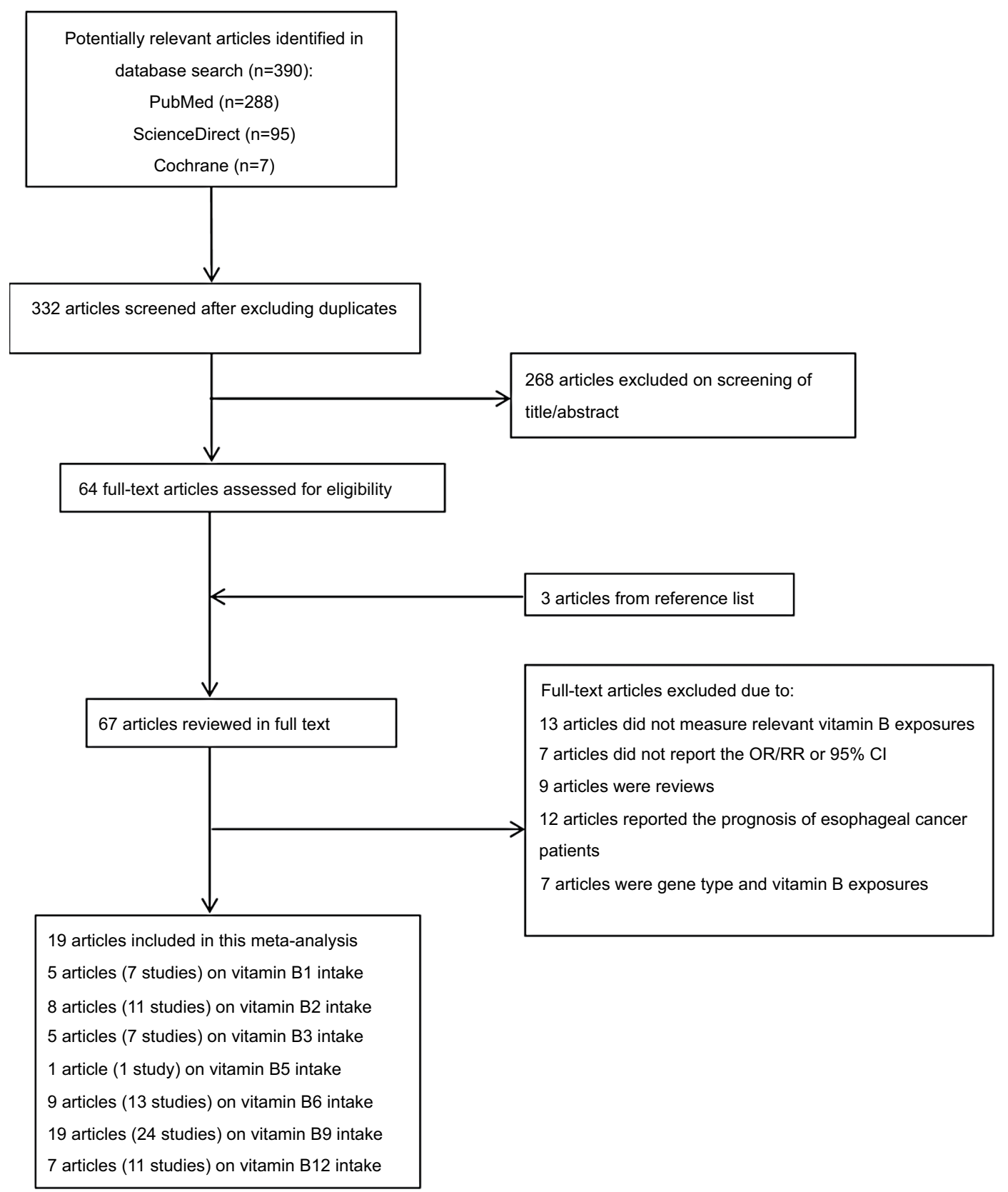

Figure I The flow diagram of screened, excluded, and analyzed publications.

reviews, 12 articles reported the prognosis of EC patients, five articles focused on gene type and vitamin B exposures, and two articles focused on blood vitamin B9, B12. As a result, 19 articles were finally selected for the meta-analysis. ${ }^{8,9,26-42}$

\section{Characteristics and quality of included studies}

We identified 19 articles in our study. Tables 1 and 2 show the main characteristics extracted from included studies. All the studies were conducted in Asia, Europe, America, and Australia and were published from 1988 to 2017. Among all the studies, one study was a cohort study ${ }^{42}$ and 18 studies were case-control studies. ${ }^{8,9,26-41}$

The quality of all studies was assessed by using the NOS scale. The overall methodological quality of articles is presented in Table 1. Overall, eleven studies had a score of $8,{ }^{26,27,30,32,33,35-40}$ four had a score of $7,{ }^{8,9,34,42}$ and the remaining studies had a score of $6 .^{28,29,36,37,39,41}$ 
Table I Characteristics of studies on B vitamin intake and esophageal cancer risk

\begin{tabular}{|c|c|c|c|c|c|c|c|c|}
\hline Author & Year & $\begin{array}{l}\text { Source of } \\
\text { control }\end{array}$ & $\begin{array}{l}\text { Study of } \\
\text { design }\end{array}$ & Country & $\begin{array}{l}\text { Cancer } \\
\text { type }\end{array}$ & Vitamin B & $\begin{array}{l}\text { Exposure } \\
\text { ascertainment }\end{array}$ & $\begin{array}{l}\text { OR }(95 \% \mathrm{CI}) \text { for } \\
\text { highest vs lowest } \\
\text { category }\end{array}$ \\
\hline Jessri et al & 2011 & $\mathrm{HB}$ & Case-control & Iran & $\mathrm{ESCC}$ & VBI & FFQ & $0.34(0.06-2.85)$ \\
\hline \multirow[t]{2}{*}{ Ibiebele et al } & 2011 & PB & Case-control & Australian & EAC & VBI & FFQ & $0.78(0.57-1.07)$ \\
\hline & & & & & ESCC & VBI & FFQ & $0.4 I(0.25-0.67)$ \\
\hline \multirow[t]{2}{*}{ Mayne et al } & 2001 & PB & Case-control & US & EAC & VBI & FFQ & $0.73(0.50-1.07)$ \\
\hline & & & & & ESCC & VBI & FFQ & $0.78(0.46-1.30)$ \\
\hline Zhang et al & 1997 & $\mathrm{HB}$ & Case-control & US & EAC & VBI & FFQ & $0.80(0.30-2.10)$ \\
\hline Brown et al & 1988 & $\mathrm{HB}$ & Case-control & US & $\mathrm{EC}$ & VBI & FFQ & $0.60(0.30-1.10)$ \\
\hline Sharp et al & 2013 & PB & Case-control & Ireland & EAC & VB2 & FFQ & $1.07(0.63-1.82)$ \\
\hline Jessri et al & 2011 & $\mathrm{HB}$ & Case-control & Iran & ESCC & VB2 & FFQ & $0.22(0.07-0.86)$ \\
\hline \multirow[t]{2}{*}{ Ibiebele et al } & 2011 & PB & Case-control & Australian & EAC & VB2 & FFQ & I.32 (0.98-I.80) \\
\hline & & & & & ESCC & VB2 & FFQ & $0.78(0.50-\mid .21)$ \\
\hline \multirow[t]{2}{*}{ Mayne et al } & 2001 & PB & Case-control & US & EAC & VB2 & FFQ & $1.11(0.82-1.52)$ \\
\hline & & & & & ESCC & VB2 & FFQ & $1.26(0.84-1.89)$ \\
\hline Bao et al & 2013 & PB & Case-control & China & ESCC & VB2 & Serum & $0.46(0.32-0.67)$ \\
\hline \multirow[t]{2}{*}{ Fanidi et al } & 2014 & PB & Nested case- & European & ESCC & VB2 & Serum & I.2I (0.54-2.72) \\
\hline & & & & & EAC & VB2 & Serum & $1.95(0.84-4.52)$ \\
\hline Zhang et al & 1997 & $\mathrm{HB}$ & Case-control & US & EAC & VB2 & Food records & $0.40(0.20-1.10)$ \\
\hline Chen et al & 2009 & PB & Case-control & US & EAC & VB2 & Validated $\mathrm{HHHQ}$ & $0.50(0.20-1.00)$ \\
\hline Jessri et al & 2011 & $\mathrm{HB}$ & Case-control & Iran & ESCC & VB3 & FFQ & $0.38(0.15-1.82)$ \\
\hline \multirow[t]{2}{*}{ Ibiebele et al } & 2011 & PB & Case-control & Australian & EAC & VB3 & FFQ & $0.71(0.52-0.96)$ \\
\hline & & & & & ESCC & VB3 & FFQ & $0.69(0.43-1.12)$ \\
\hline \multirow[t]{2}{*}{ Mayne et al } & 2001 & PB & Case-control & US & EAC & VB3 & FFQ & I.07 (0.77-I.48) \\
\hline & & & & & ESCC & VB3 & FFQ & $0.74(0.48-1.16)$ \\
\hline Zhang et al & 1997 & $\mathrm{HB}$ & Case-control & US & EAC & VB3 & $\mathrm{FFQ}$ & $0.20(0.10-0.70)$ \\
\hline Chen et al & 2009 & PB & Case-control & US & EAC & VB3 & Validated $\mathrm{HHHQ}$ & $0.80(0.40-1.50)$ \\
\hline Jessri et al & 2011 & $H B$ & Case-control & Iran & ESCC & VB5 & FFQ & $0.49(0.35-2.08)$ \\
\hline Sharp et al & 2013 & PB & Case-control & Ireland & EAC & VB6 & FFQ & $0.37(0.22-0.63)$ \\
\hline Jessri et al & 2011 & $\mathrm{HB}$ & Case-control & Iran & ESCC & VB6 & FFQ & $0.17(0.05-0.91)$ \\
\hline \multirow[t]{2}{*}{ Ibiebele et al } & 2011 & PB & Case-control & Australian & EAC & VB6 & FFQ & $0.53(0.39-0.74)$ \\
\hline & & & & & ESCC & VB6 & FFQ & $0.66(0.42-1.05)$ \\
\hline \multirow[t]{2}{*}{ Xiao et al } & 2014 & PB & cohort & US & ESCC & VB6 & FFQ & $0.86(0.5 \mathrm{I}-\mathrm{I} .45)$ \\
\hline & & & & & EAC & VB6 & FFQ & $1.00(0.76-1.32)$ \\
\hline \multirow[t]{2}{*}{ Mayne et al } & 2001 & PB & Case-control & US & EAC & VB6 & FFQ & $0.53(0.38-0.73)$ \\
\hline & & & & & ESCC & VB6 & FFQ & $0.45(0.30-0.69)$ \\
\hline \multirow[t]{2}{*}{ Fanidi et al } & 2014 & PB & $\begin{array}{l}\text { Nested Case- } \\
\text { control }\end{array}$ & European & ESCC & VB6 & Serum & $2.26(1.06-4.84)$ \\
\hline & & & & & EAC & VB6 & Serum & $0.63(0.30-1.33)$ \\
\hline Galeone et al & 2006 & $\mathrm{HB}$ & Case-control & $\begin{array}{l}\text { Italy and } \\
\text { Swiss }\end{array}$ & ESCC & VB6 & FFQ & $0.99(0.60-1.31)$ \\
\hline Zhang et al & 1997 & $\mathrm{HB}$ & Case-control & US & EAC & VB6 & FFQ & $0.20(0.10-0.70)$ \\
\hline Chen et al & 2009 & PB & Case-control & US & EAC & VB6 & Validated $\mathrm{HHHQ}$ & $0.7(0.30-1.30)$ \\
\hline Ling & 2013 & PB & Case-control & China & ESCC & VB9 & Serum & $0.11(0.04-0.33)$ \\
\hline Sharp et al & 2013 & PB & Case-control & Ireland & EAC & VB9 & FFQ & $0.52(0.30-0.89)$ \\
\hline Zhao et al & 2011 & $\mathrm{HB}$ & Case-control & China & ESCC & VB9 & FFQ & $0.61(0.36-1.07)$ \\
\hline
\end{tabular}




\begin{tabular}{|c|c|c|}
\hline $\begin{array}{l}\text { Participants } \\
\text { (cases) }\end{array}$ & Adjust variables & $\begin{array}{l}\text { New Castle- } \\
\text { Ottawa scale }\end{array}$ \\
\hline $144(48)$ & $\begin{array}{l}\text { Age, gender, energy intake, BMI, smoking status, physical activity, education level, gastroesophageal reflux } \\
\text { disease symptoms }\end{array}$ & 8 \\
\hline $519(147)$ & Age, gender, education, BMI, alcohol intake, smoking status, energy intake, NSAID use & 8 \\
\hline $429(57)$ & Age, gender, education, BMI, alcohol intake, smoking status, energy intake, NSAID use & 8 \\
\hline $969(282)$ & Age, gender, site, race, proxy status, income, education, BMl, smoking status, alcohol, energy intake & 8 \\
\hline $893(206)$ & Age, gender, site, race, proxy status, income, education, BMI, smoking status, alcohol, energy intake & 8 \\
\hline $48(18)$ & NR & 6 \\
\hline $629(207)$ & Smoking status, alcohol intake & 6 \\
\hline $129(64)$ & Age, gender, total energy intake & 9 \\
\hline $144(48)$ & $\begin{array}{l}\text { Age, gender, energy intake, BMI, smoking status, physical activity, education level, gastroesophageal reflux } \\
\text { disease symptoms }\end{array}$ & 8 \\
\hline $518(146)$ & Age, gender, education, BMI, alcohol intake, smoking status, energy intake, NSAID use & 8 \\
\hline $422(50)$ & Age, gender, education, BMI, alcohol intake, smoking status, energy intake, NSAID use & 8 \\
\hline $969(282)$ & Age, gender, site, race, proxy status, income, education, BMI, smoking status, alcohol, energy intake & 8 \\
\hline $893(206)$ & Age, gender, site, race, proxy status, income, education, BMI, smoking status, alcohol, energy intake & 8 \\
\hline $212(106)$ & Age, gender, site & 7 \\
\hline $252(123)$ & Age, gender, country, educational attainment, smoking status, alcohol intake & 8 \\
\hline $268(26)$ & Age, gender, country, educational attainment, smoking status, alcohol intake & 8 \\
\hline $44(13)$ & NR & 6 \\
\hline $573(124)$ & $\begin{array}{l}\text { Age, gender, respondent type, BMI, alcohol intake, tobacco use, education level, family history, vitamin } \\
\text { supplement use }\end{array}$ & 8 \\
\hline $144(48)$ & $\begin{array}{l}\text { Age, gender, energy intake, BMI, smoking status, physical activity, education level, gastroesophageal reflux } \\
\text { disease symptoms }\end{array}$ & 8 \\
\hline $515(143)$ & Age, gender, education, BMI, alcohol intake, smoking status, energy intake, NSAID use & 8 \\
\hline $421(49)$ & Age, gender, education, BMI, alcohol intake, smoking status, energy intake, NSAID use & 8 \\
\hline $969(282)$ & Age, gender, site, race, proxy status, income, education, BMI, smoking status, alcohol, energy intake & 8 \\
\hline $893(206)$ & Age, gender, site, race, proxy status, income, education, BMI, smoking status, alcohol, energy intake & 8 \\
\hline $44(13)$ & NR & 6 \\
\hline $573(124)$ & $\begin{array}{l}\text { Age, gender, respondent type, BMI, alcohol intake, tobacco use, education level, family history, vitamin } \\
\text { supplement use }\end{array}$ & 8 \\
\hline $144(48)$ & $\begin{array}{l}\text { Age, gender, energy intake, BMI, smoking status, physical activity, education level, gastroesophageal reflux } \\
\text { disease symptoms }\end{array}$ & 8 \\
\hline $142(46)$ & Age, gender, total energy intake & 9 \\
\hline 145 (49) & $\begin{array}{l}\text { Age, gender, energy intake, BMI, smoking status, physical activity, education level, gastroesophageal reflux } \\
\text { disease symptoms }\end{array}$ & 8 \\
\hline $517(146)$ & Age, gender, education, BMI, alcohol intake, smoking status, energy intake, NSAID use & 8 \\
\hline $423(52)$ & Age, gender, education, BMI, alcohol intake, smoking status, energy intake, NSAID use & 8 \\
\hline $4,471,303(25)$ & $\begin{array}{l}\text { Age, gender, race, education, marital status, health status, BMI, smoking status, alcohol, vigorous physical } \\
\text { activity, multivitamin use, family history of cancer, energy intake }\end{array}$ & 7 \\
\hline 4,47I,303 (98) & $\begin{array}{l}\text { Age, gender, race, education, marital status, health status, BMI, smoking status, alcohol, vigorous physical } \\
\text { activity, multivitamin use, family history of cancer, energy intake }\end{array}$ & 7 \\
\hline $969(282)$ & Age, gender, site, race, proxy status, income, education, BMI, smoking status, alcohol, energy intake & 8 \\
\hline $893(206)$ & Age, gender, site, race, proxy status, income, education, BMI, smoking status, alcohol, energy intake & 8 \\
\hline $257(128)$ & Age, gender, country, educational attainment, smoking status, alcohol intake & 8 \\
\hline $270(16)$ & Age, gender, country, educational attainment, smoking status, alcohol intake & 8 \\
\hline $405(108)$ & Age, center, education, BMI, smoking, alcohol drinking & 7 \\
\hline $44(13)$ & NR & 6 \\
\hline $573(124)$ & $\begin{array}{l}\text { Age, gender, respondent type, BMI, alcohol intake, tobacco use, education level, family history, vitamin } \\
\text { supplement use }\end{array}$ & 8 \\
\hline $48(6)$ & Age, gender, smoking habit, drinking & 8 \\
\hline $136(55)$ & Age, gender, total energy intake & 8 \\
\hline $174(52)$ & Age, gender & 6 \\
\hline
\end{tabular}


Table I (Continued)

\begin{tabular}{|c|c|c|c|c|c|c|c|c|}
\hline Author & Year & $\begin{array}{l}\text { Source of } \\
\text { control }\end{array}$ & $\begin{array}{l}\text { Study of } \\
\text { design }\end{array}$ & Country & $\begin{array}{l}\text { Cancer } \\
\text { type }\end{array}$ & Vitamin B & $\begin{array}{l}\text { Exposure } \\
\text { ascertainment }\end{array}$ & $\begin{array}{l}\text { OR }(95 \% \mathrm{Cl}) \text { for } \\
\text { highest vs lowest } \\
\text { category }\end{array}$ \\
\hline Jessri et al & 2011 & $\mathrm{HB}$ & Case-control & Iran & ESCC & VB9 & FFQ & $0.08(0.02-0.90)$ \\
\hline Chang et al & 2015 & PB & Case-control & China & EC & VB9 & Plasma & $1.58(0.95-2.64)$ \\
\hline \multirow[t]{2}{*}{ Ibiebele et al } & 2011 & PB & Case-control & Australian & EAC & VB9 & FFQ & $0.72(0.53-0.98)$ \\
\hline & & & & & ESCC & VB9 & FFQ & $0.78(0.5 \mathrm{I}-\mathrm{I} .19)$ \\
\hline Aune et al & 2011 & $\mathrm{HB}$ & Case-control & Uruguay & $\mathrm{EC}$ & VB9 & FFQ & $0.29(0.14-0.60)$ \\
\hline \multirow[t]{2}{*}{ Xiao et al } & 2014 & PB & cohort & US & ESCC & VB9 & FFQ & $1.07(0.59-1.94)$ \\
\hline & & & & & EAC & VB9 & FFQ & $1.00(0.76-\mid .31)$ \\
\hline \multirow[t]{2}{*}{ Mayne et al } & 2001 & PB & Case-control & US & EAC & VB9 & FFQ & $0.48(0.36-0.66)$ \\
\hline & & & & & ESCC & VB9 & FFQ & $0.58(0.39-0.86)$ \\
\hline Bao et al & 2013 & PB & Case-control & China & ESCC & VB9 & Serum & $0.43(0.29-0.62)$ \\
\hline \multirow[t]{2}{*}{ Fanidi et al } & 2014 & PB & $\begin{array}{l}\text { Nested case- } \\
\text { control }\end{array}$ & European & ESCC & VB9 & Serum & $1.03(0.47-2.24)$ \\
\hline & & & & & EAC & VB9 & Serum & $1.68(0.79-3.56)$ \\
\hline Galeone et al & 2006 & $\mathrm{HB}$ & Case-control & $\begin{array}{l}\text { Italy and } \\
\text { Swiss }\end{array}$ & ESCC & VB9 & FFQ & $0.68(0.46-1.00)$ \\
\hline Tavani et al & 2012 & $\mathrm{HB}$ & Case-control & Italy & EC & VB9 & FFQ & $0.26(0.14-0.48)$ \\
\hline \multirow{2}{*}{$\begin{array}{l}\text { Bollschweil } \\
\text { et al }\end{array}$} & 2002 & PB & Case-control & Germany & EAC & VB9 & FFQ & $5.00(2.10-13.60)$ \\
\hline & & & & & ESCC & VB9 & FFQ & $3.20(1.30-9.10)$ \\
\hline Zhang et al & 1997 & $\mathrm{HB}$ & Case-control & US & EAC & VB9 & FFQ & $0.70(0.30-1.70)$ \\
\hline Qin et al & 2008 & $\mathrm{HB}$ and $\mathrm{PB}$ & Case-control & China & EC & VB9 & FFQ & $0.52(0.33-0.82)$ \\
\hline Brown et al & 1988 & $\mathrm{HB}$ & Case-control & US & $\mathrm{EC}$ & VB9 & FFQ & $0.70(0.40-1.30)$ \\
\hline Chen et al & 2009 & PB & Case-control & US & EAC & VB9 & $\mathrm{HHHQ}$ & $0.50(0.30-1.00)$ \\
\hline Yang et al & 2005 & $\mathrm{HB}$ & Case-control & Japan & EC & VB9 & SQFFQ & $0.77(0.45-1.3 \mathrm{I})$ \\
\hline Sharp et al & 2013 & PB & Case-control & Ireland & EAC & VBI 2 & FFQ & $3.87(2.22-6.73)$ \\
\hline Jessri et al & 2011 & $\mathrm{HB}$ & Case-control & Iran & ESCC & VBI 2 & FFQ & $1.33(0.60-3.03)$ \\
\hline Chang et al & 2015 & PB & Case-control & China & $\mathrm{EC}$ & VBI2 & Plasma & $3.07(1.73-5.45)$ \\
\hline \multirow[t]{2}{*}{ Ibiebele et al } & 2011 & PB & Case-control & Australian & EAC & VBI2 & FFQ & $0.96(0.7 I-1.30)$ \\
\hline & & & & & ESCC & VBI2 & FFQ & $0.89(0.58-1.32)$ \\
\hline \multirow[t]{2}{*}{ Xiao et al } & 2014 & PB & cohort & US & ESCC & VBI2 & FFQ & $0.85(0.52-I .4 I)$ \\
\hline & & & & & EAC & VBI2 & FFQ & $1.04(0.80-1.34)$ \\
\hline \multirow[t]{2}{*}{ Mayne et al } & 2001 & PB & Case-control & US & EAC & VBI2 & FFQ & $1.39(1.10-1.76)$ \\
\hline & & & & & ESCC & VBI2 & FFQ & $1.51(1.15-2.00)$ \\
\hline \multirow[t]{2}{*}{ Fanidi et al } & 2014 & PB & Nested case- & European & ESCC & VBI 2 & Serum & $\mathrm{I} .07(0.5 \mathrm{I}-2.23)$ \\
\hline & & & & & EAC & VBI2 & Serum & $1.17(0.56-2.44)$ \\
\hline
\end{tabular}

Abbreviations: BMI, body mass index; EAC, esophageal adenocarcinoma; EC, esophageal carcinoma; ESCC, esophageal squamous cell carcinoma; HB, hospital-based; N/A, not available; NR, not reported; NSAID, nonsteroidal anti-inflammatory drug; PB, population-based; VB, vitamin B; FFQ, food frequency questionnaires; HHQ, health habits and history questionnaires.

\section{Multivitamin B intake}

Our results showed a statistically significant inverse association between use of multivitamin B supplements and EC (OR=0.70; 95\% CI: 0.59-0.83). There was statistically significant heterogeneity among all the studies $\left(I^{2}=77.9 \%\right.$; $P=0.00)$.

\section{Subgroup analysis of the source of the control group}

Subgroup analysis of the source of the control group showed that dietary vitamin $\mathrm{B}$ was a protective factor for $\mathrm{EC}$ in both subgroups (hospital-based: OR $=0.575,95 \%$ CI: $0.492-0.672$; population-based: $\mathrm{OR}=0.868,95 \% \mathrm{CI}: 0.820-0.919$ ). 


\begin{tabular}{|c|c|c|}
\hline $\begin{array}{l}\text { Participants } \\
\text { (cases) }\end{array}$ & Adjust & $\begin{array}{l}\text { New Castle- } \\
\text { Ottawa scale }\end{array}$ \\
\hline $144(48)$ & $\begin{array}{l}\text { Age, gender, energy intake, BMI, smoking status, physical activity, education level, gastroesophageal reflux } \\
\text { disease symptoms }\end{array}$ & 8 \\
\hline $178(75)$ & Age, gender, BMI, education, smoking status, alcohol drinking frequency & 8 \\
\hline $491(117)$ & Age, gender, education, BMI, alcohol intake, smoking status, energy intake, NSAID use & 8 \\
\hline $430(56)$ & Age, gender, education, BMI, alcohol intake, smoking status, energy intake, NSAID use & 8 \\
\hline $2,102(70)$ & $\begin{array}{l}\text { Age, gender, residence, education, income, interviewer, smoking status, alcohol, dietary fiber, iron, BMI, } \\
\text { energy intake }\end{array}$ & 7 \\
\hline $447 \mid 303(2 \mid)$ & $\begin{array}{l}\text { Age, gender, race, education, marital status, health status, BMI, smoking status, alcohol, vigorous physical } \\
\text { activity, multivitamin use, family history of cancer, energy intake }\end{array}$ & 7 \\
\hline $447 \mid 303(98)$ & $\begin{array}{l}\text { Age, gender, race, education, marital status, health status, BMI, smoking status, alcohol, vigorous physical } \\
\text { activity, multivitamin use, family history of cancer, energy intake }\end{array}$ & 7 \\
\hline $969(282)$ & Age, gender, site, race, proxy status, income, education, BMI, smoking status, alcohol, energy intake & 8 \\
\hline $893(206)$ & Age, gender, site, race, proxy status, income, education, BMI, smoking status, alcohol, energy intake & 8 \\
\hline $212(106)$ & Age, gender, site & 7 \\
\hline $255(126)$ & Age, gender, country, educational attainment, smoking status, alcohol intake & 8 \\
\hline $274(26)$ & Age, gender, country, educational attainment, smoking status, alcohol intake & 8 \\
\hline $404(90)$ & Age, center, education, BMI, smoking, alcohol drinking & 7 \\
\hline $443(128)$ & $\begin{array}{l}\text { Age, gender, study center, year of interview, education, alcohol drinking, tobacco smoking, BMI, energy } \\
\text { intake, physical activity }\end{array}$ & 6 \\
\hline $38(25)$ & NR & 6 \\
\hline $29(16)$ & NR & 6 \\
\hline $49(18)$ & NR & 6 \\
\hline $360(120)$ & NR & 5 \\
\hline $629(207)$ & Smoking status, alcohol intake & 6 \\
\hline $573(124)$ & $\begin{array}{l}\text { Age, gender, respondent type, BMl, alcohol intake, tobacco use, education level, family history, vitamin } \\
\text { supplement use }\end{array}$ & 8 \\
\hline $270(62)$ & Smoking status, alcohol intake, total energy intake & 6 \\
\hline $124(81)$ & Age, gender, total energy & 8 \\
\hline $143(47)$ & $\begin{array}{l}\text { Age, gender, energy intake, BMI, smoking status, physical activity, education level, gastroesophageal reflux } \\
\text { disease symptoms }\end{array}$ & 8 \\
\hline $195(93)$ & Age, gender, BMI, education, smoking status, alcohol drinking frequency & 8 \\
\hline $528(155)$ & Age, gender, education, BMI, alcohol intake, smoking status, energy intake, NSAID use & 8 \\
\hline $438(65)$ & Age, gender, education, BMI, alcohol intake, smoking status, energy intake, NSAID use & 8 \\
\hline $447 \mid 303(28)$ & $\begin{array}{l}\text { Age, gender, race, education, marital status, health status, BMl, smoking status, alcohol, vigorous physical } \\
\text { activity, multivitamin use, family history of cancer, energy intake }\end{array}$ & 7 \\
\hline $447 \mid 303(123)$ & $\begin{array}{l}\text { Age, gender, race, education, marital status, health status, BMl, smoking status, alcohol, vigorous physical } \\
\text { activity, multivitamin use, family history of cancer, energy intake }\end{array}$ & 7 \\
\hline $969(282)$ & Age, gender, site, race, proxy status, income, education, BMI, smoking status, alcohol, energy intake & 8 \\
\hline $893(206)$ & Age, gender, site, race, proxy status, income, education, BMl, smoking status, alcohol, energy intake & 8 \\
\hline $274(145)$ & Age, gender, country, educational attainment, smoking status, alcohol intake & 8 \\
\hline $298(18)$ & Age, gender, country, educational attainment, smoking status, alcohol intake & 8 \\
\hline
\end{tabular}

\section{Subgroup analysis of EC pathological types}

Subgroup analysis based on EC pathological types showed that dietary vitamin $\mathrm{B}$ was protective against esophageal squamous cell carcinoma $(\mathrm{OR}=0.762,95 \% \mathrm{CI}$ : $0.697-0.833)$ and esophageal adenocarcinoma $(\mathrm{OR}=0.870,95 \% \mathrm{CI}: 0.811-0.933)$.

\section{Vitamin BI intake}

The association between vitamin B1 intake and EC risk was examined in seven case-control studies. The multivariable adjusted ORs for each study and combination of all studies for the highest vs lowest level of dietary vitamin B1 intake are shown in Figure 2. The pooled OR of EC for the highest 
Table 2 Characteristics of studies on B vitamin intake

\begin{tabular}{|c|c|c|c|c|}
\hline Author & Year & Vitamin B & $\begin{array}{l}\text { Exposure } \\
\text { ascertainment }\end{array}$ & Highest vs lowest category \\
\hline Jessri et al & 2011 & VBI & FFQ & - \\
\hline \multirow[t]{2}{*}{ Ibiebele et al } & 2011 & VBI & FFQ & $0.4-1.5$ vs $2.1-5.8$ (mg/d) \\
\hline & & VBI & FFQ & $0.4-1.5$ vs $2.1-5.8(\mathrm{mg} / \mathrm{d})$ \\
\hline \multirow[t]{2}{*}{ Mayne et al } & 2001 & VBI & FFQ & - \\
\hline & & VBI & FFQ & - \\
\hline Zhang et al & 1997 & VBI & FFQ & - \\
\hline Brown et al & 1988 & VBI & FFQ & - \\
\hline Sharp et al & 2013 & VB2 & FFQ & $\leq 1.8 \mathrm{vs} \geq 2.8 \mathrm{mg}(\mathrm{mg} / \mathrm{d})$ \\
\hline Jessri et al & 2011 & VB2 & FFQ & - \\
\hline \multirow[t]{2}{*}{ Ibiebele et al } & 2011 & VB2 & FFQ & $0.5-1.8$ vs $2.7-7.1$ (mg/d) \\
\hline & & VB2 & FFQ & $0.5-1.8$ vs $2.7-7.1(\mathrm{mg} / \mathrm{d})$ \\
\hline \multirow[t]{2}{*}{ Mayne et al } & 2001 & VB2 & FFQ & - \\
\hline & & VB2 & FFQ & - \\
\hline Bao et al & 2013 & VB2 & Serum & $<2,401.86$ vs $>2845.42(\mu \mathrm{g} / \mathrm{L})$ \\
\hline \multirow[t]{2}{*}{ Fanidi et al } & 2014 & VB2 & Serum & $2.5-9.4$ vs $21.4-199(\mathrm{nmol} / \mathrm{L})$ \\
\hline & & VB2 & Serum & $2.5-9.4$ vs $21.4-199$ (nmol/L) \\
\hline Zhang et al & 1997 & VB2 & Food records & - \\
\hline Chen et al & 2009 & VB2 & Validated $\mathrm{HHHQ}$ & - \\
\hline Jessri et al & 2011 & VB3 & FFQ & - \\
\hline \multirow[t]{2}{*}{ lbiebele et al } & 2011 & VB3 & FFQ & $28-50 \mathrm{mg}$ \\
\hline & & VB3 & FFQ & $28-50 \mathrm{mg}$ \\
\hline \multirow[t]{2}{*}{ Mayne et al } & 2001 & VB3 & FFQ & - \\
\hline & & VB3 & FFQ & - \\
\hline Zhang et al & 1997 & VB3 & FFQ & - \\
\hline Chen et al & 2009 & VB3 & Validated HHHQ & - \\
\hline Jessri et al & 2011 & VB5 & FFQ & - \\
\hline Sharp et al & 2013 & VB6 & FFQ & $\leq 2.3$ vs $\geq 3.2(\mathrm{mg} / \mathrm{d})$ \\
\hline Jessri et al & 2011 & VB6 & FFQ & - \\
\hline \multirow[t]{2}{*}{ Ibiebele et al } & 2011 & VB6 & FFQ & $0.3-1.1$ vs $1.5-3.0(\mathrm{mg} / \mathrm{d})$ \\
\hline & & VB6 & FFQ & $0.3-I . I$ vs $1.5-3.0(\mathrm{mg} / \mathrm{d})$ \\
\hline \multirow[t]{2}{*}{ Xiao et al } & 2014 & VB6 & FFQ & - \\
\hline & & VB6 & FFQ & - \\
\hline \multirow[t]{2}{*}{ Mayne et al } & 2001 & VB6 & FFQ & - \\
\hline & & VB6 & FFQ & - \\
\hline \multirow[t]{2}{*}{ Fanidi et al } & 2014 & VB6 & Serum & $7.2-25.6$ vs $47.7-272(\mathrm{nmol} / \mathrm{L})$ \\
\hline & & VB6 & Serum & $7.2-25.6$ vs $47.7-272(\mathrm{nmol} / \mathrm{L})$ \\
\hline Galeone et al & 2006 & VB6 & FFQ & - \\
\hline Zhang et al & 1997 & VB6 & FFQ & - \\
\hline Chen et al & 2009 & VB6 & Validated HHHQ & - \\
\hline Ling & 2013 & VB9 & Serum & $<17.04$ vs $>34.19(\mu \mathrm{g} / \mathrm{L})$ \\
\hline Sharp et al & 2013 & VB9 & FFQ & $\leq 318$ vs $\geq 421(\mu g / d)$ \\
\hline Zhao et al & 2011 & VB9 & FFQ & $<230$ vs $>300(\mu \mathrm{g} / \mathrm{d})$ \\
\hline Jessri et al & 2011 & VB9 & FFQ & - \\
\hline Chang et al & 2015 & VB9 & Plasma & $\leq 8.90$ vs $>17.66(\mathrm{nmol} / \mathrm{L})$ \\
\hline \multirow[t]{2}{*}{ Ibiebele et al } & 2011 & VB9 & FFQ & $42-230$ vs $336-673(\mu \mathrm{g} / \mathrm{d})$ \\
\hline & & VB9 & FFQ & $42-230$ vs $336-673(\mu \mathrm{g} / \mathrm{d})$ \\
\hline Aune et al & 2011 & VB9 & FFQ & - \\
\hline \multirow[t]{2}{*}{ Xiao et al } & 2014 & VB9 & FFQ & - \\
\hline & & VB9 & FFQ & - \\
\hline \multirow[t]{2}{*}{ Mayne et al } & 2001 & VB9 & FFQ & - \\
\hline & & VB9 & FFQ & - \\
\hline Bao et al & 2013 & VB9 & Serum & $<28.27$ vs $>35.06(\mu \mathrm{g} / \mathrm{L})$ \\
\hline \multirow{2}{*}{ Fanidi et al } & 2014 & VB9 & Serum & $0.3-9.1$ to $-18.2-109(\mathrm{nmol} / \mathrm{L})$ \\
\hline & & VB9 & Serum & $0.3-9.1$ to $-18.2-109(\mathrm{nmol} / \mathrm{L})$ \\
\hline Galeone et al & 2006 & VB9 & FFQ & - \\
\hline Tavani et al & 2012 & VB9 & FFQ & $<208.77$ vs $>312.47(\mu \mathrm{g} / \mathrm{d})$ \\
\hline
\end{tabular}

(Continued) 
Table 2 (Continued)

\begin{tabular}{|c|c|c|c|c|}
\hline Author & Year & Vitamin B & $\begin{array}{l}\text { Exposure } \\
\text { ascertainment }\end{array}$ & Highest vs lowest category \\
\hline \multirow[t]{2}{*}{ Bollschweiler et al } & 2002 & VB9 & FFQ & $0-164(\mu g / d)$ \\
\hline & & VB9 & FFQ & $0-164(\mu \mathrm{g} / \mathrm{d})$ \\
\hline Zhang et al & 1997 & VB9 & FFQ & - \\
\hline Qin et al & 2008 & VB9 & FFQ & - \\
\hline Brown et al & 1988 & VB9 & FFQ & - \\
\hline Chen et al & 2009 & VB9 & $\mathrm{HHHQ}$ & - \\
\hline Yang et al & 2005 & VB9 & SQFFQ & $<300$ vs $>400(\mu \mathrm{g} / \mathrm{d})$ \\
\hline Sharp et al & 2013 & VBI2 & FFQ & $\leq 6.4$ vs $\geq 9.7(\mu \mathrm{g} / \mathrm{d})$ \\
\hline Jessri et al & 2011 & VBI2 & FFQ & - \\
\hline Chang et al & 2015 & VBI2 & Plasma & $\leq 154.23$ vs $>324.06(\mathrm{pmol} / \mathrm{L})$ \\
\hline \multirow[t]{2}{*}{ Ibiebele et al } & 2011 & VBI2 & FFQ & $0-I . I$ vs $2.1-7.8(\mu \mathrm{g} / \mathrm{d})$ \\
\hline & & VBI2 & FFQ & $0-1.1$ vs $2.1-7.8(\mu \mathrm{g} / \mathrm{d})$ \\
\hline \multirow[t]{2}{*}{ Xiao et al } & 2014 & VBI2 & FFQ & - \\
\hline & & VBI2 & FFQ & - \\
\hline \multirow[t]{2}{*}{ Mayne et al } & 2001 & VBI2 & FFQ & - \\
\hline & & VBI2 & FFQ & - \\
\hline \multirow[t]{2}{*}{ Fanidi et al } & 2014 & VBI2 & Serum & $\begin{array}{l}75.1-265 \text { vs } 392-2,737 \\
\text { (pmol/L) }\end{array}$ \\
\hline & & VBI2 & Serum & $\begin{array}{l}75.1-265 \text { vs } 392-2,737 \\
\text { (pmol/L) }\end{array}$ \\
\hline
\end{tabular}

Abbreviations: VB, vitamin B; FFQ, food frequency questionnaires; $\mathrm{HHHQ}$, health habits and history questionnaires; SQFFQ, semi-quantitative food frequency questionnaires.

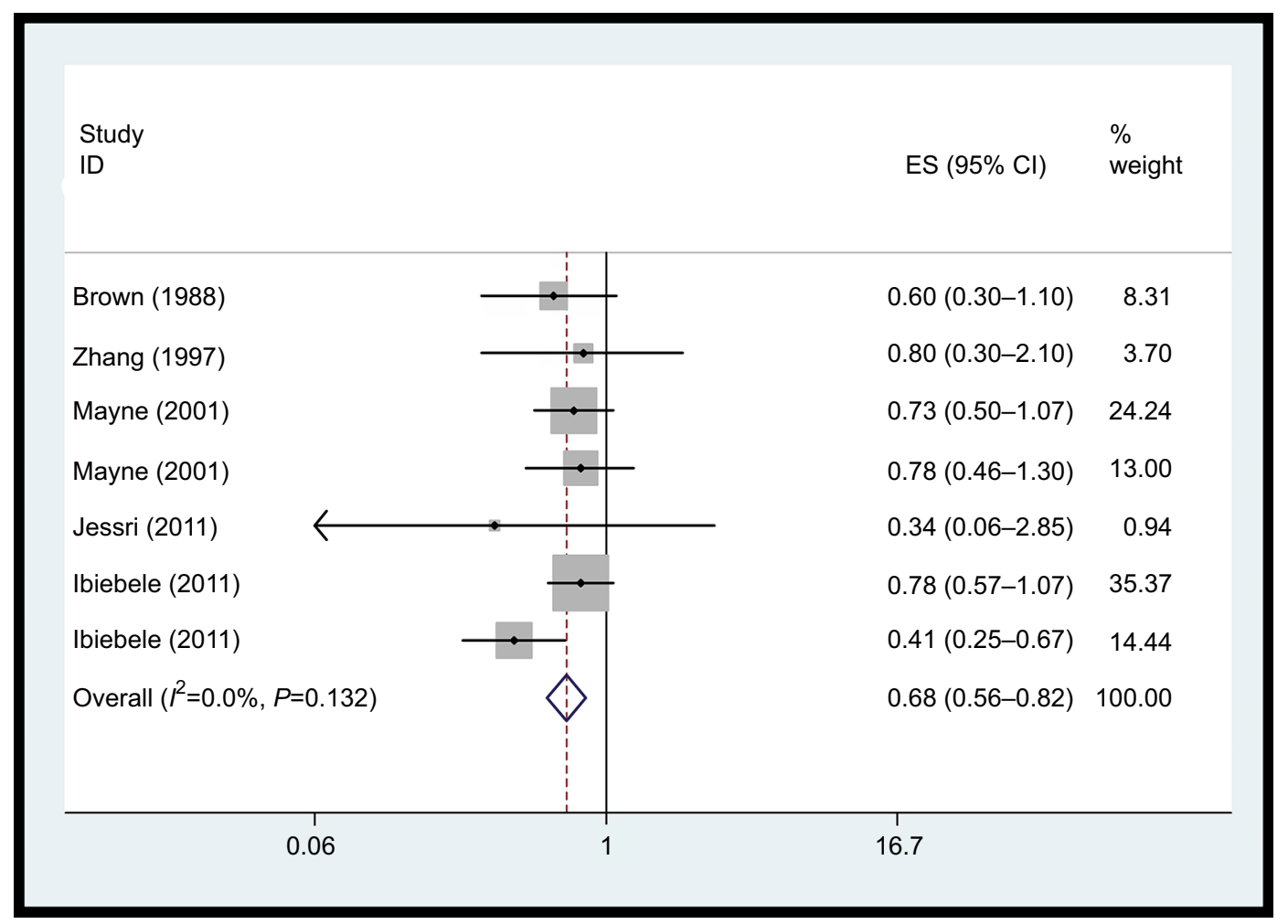

Figure 2 Forest plot between highest vs lowest categories of vitamin $\mathrm{BI}$ intake and EC risk. Abbreviation: EC, esophageal cancer.

vs lowest level of vitamin B1 intake was 0.68 (95\% CI: 0.56$0.82)$. No heterogeneity was detected $\left(I^{2}=0.0 \%, P=0.432\right)$. It was not possible to perform dose-response meta-analyses due to limited data.

\section{Vitamin B2 intake}

We did not observe a statistically significant association for vitamin $\mathrm{B} 2$ supplements and $\mathrm{EC}$ risk (Figure 3, $\mathrm{OR}=0.86$; 95\% CI: 0.64-1.16) based on eleven studies. There was 


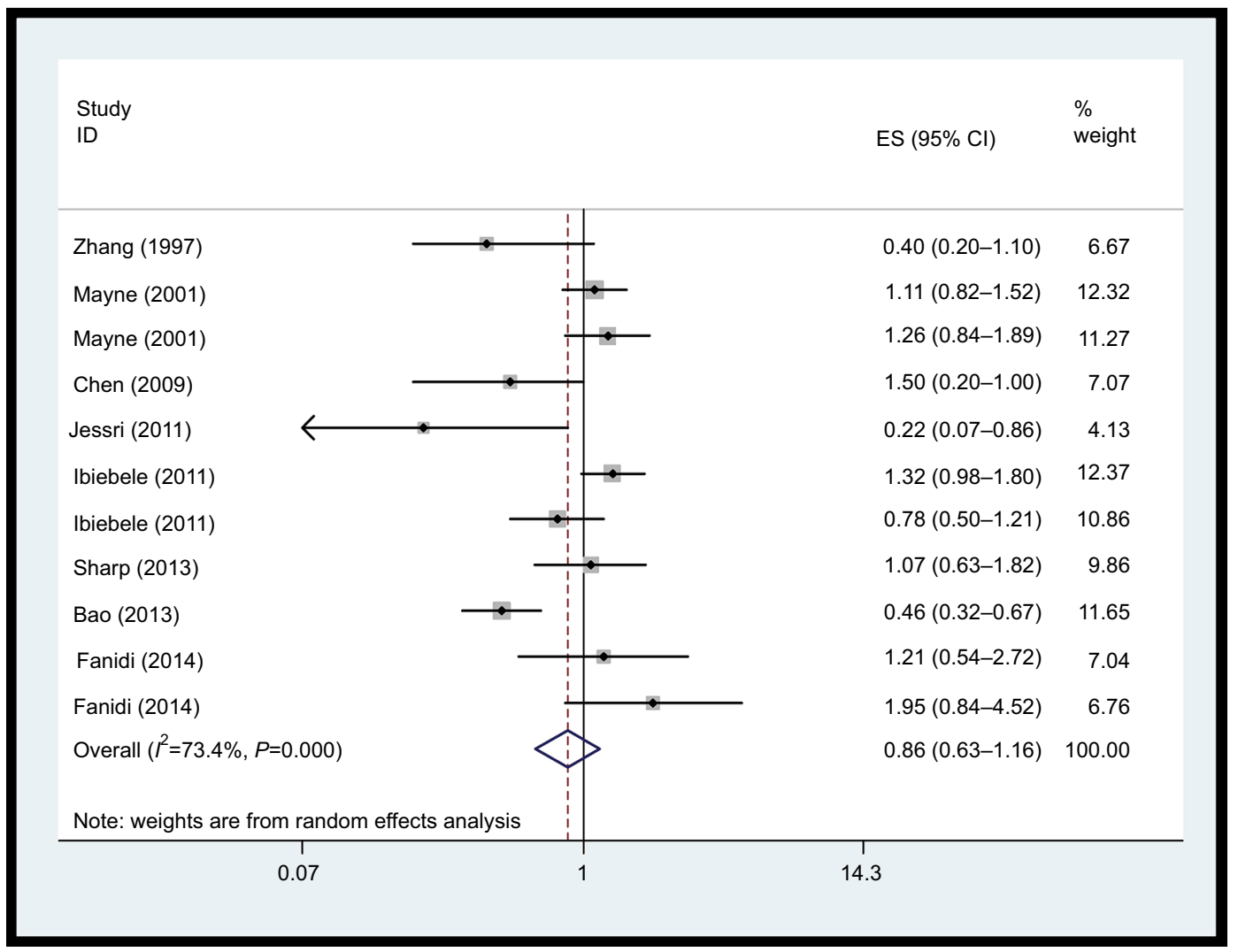

Figure 3 Forest plot between highest vs lowest categories of vitamin B2 intake and esophageal cancer risk. Abbreviation: ES, esophageal squamous carcinoma.

statistically significant heterogeneity among the studies on dietary vitamin B2 intake $\left(I^{2}=70.2 \% ; P<0.001\right)$.

\section{Vitamin B3 intake}

As shown in Figure 4, seven studies examined the association between vitamin $\mathrm{B} 3$ intake and $\mathrm{EC}$ risk. The pooled OR for the highest vs lowest vitamin B3 intake was $0.70(95 \%$ CI: $\left.0.53-0.94, I^{2}=53.9 \%, P=0.043\right)$. Dose-response metaanalyses were not done due to data limitations.

\section{Vitamin B5 intake}

There was only one study which showed the association between vitamin $\mathrm{B} 5$ intake and $\mathrm{EC}$ risk $(\mathrm{OR}=0.49,95 \%$ CI:0.20-1.20), suggesting that vitamin B5 intake was not significantly associated with the risk of EC.

\section{Vitamin B6 intake}

A total of 13 studies assessed the association between dietary vitamin B6 intake and EC risk. Figure 5 shows that the pooled OR of EC risk for the highest vs the lowest categories of vitamin B6 intake was 0.64 (95\% CI: $0.49-0.83, I^{2}=73.0 \%$, $P=0.00$ ), indicating that vitamin $\mathrm{B} 6$ intake had a protective effect against EC risk. For an increase of $100 \mu \mathrm{g} /$ day of dietary vitamin B6 intake, a statistically significant, inverse association with EC risk (OR=0.98, 95\% CI: 0.98-0.99) was detected.

\section{Vitamin B9 intake}

The association between dietary folate intake and EC risk was examined in 15 studies. The multivariable adjusted ORs for each study and combination of all studies for the highest vs lowest level of dietary folate intake are shown in Figure 6. The pooled OR of EC for the highest vs lowest level of dietary folate intake was 0.63 (95\% CI: 0.56-0.71). There was statistically significant heterogeneity among the studies on dietary folate intake $\left(I^{2}=70.2 \% ; P=0.00\right)$. Dose-response meta-analysis was based on seven studies. A statistically significant, inverse association was observed for an increase of $100 \mu \mathrm{g} /$ day in supplemental vitamin B9 and $\mathrm{EC}$ risk (OR=0.89; 95\% CI: 0.86-0.94).

\section{Vitamin BI 2 intake}

Inconsistent associations were observed for use of vitamin B12 supplements and EC risk in our study $(\mathrm{OR}=1.34,95 \%$ 


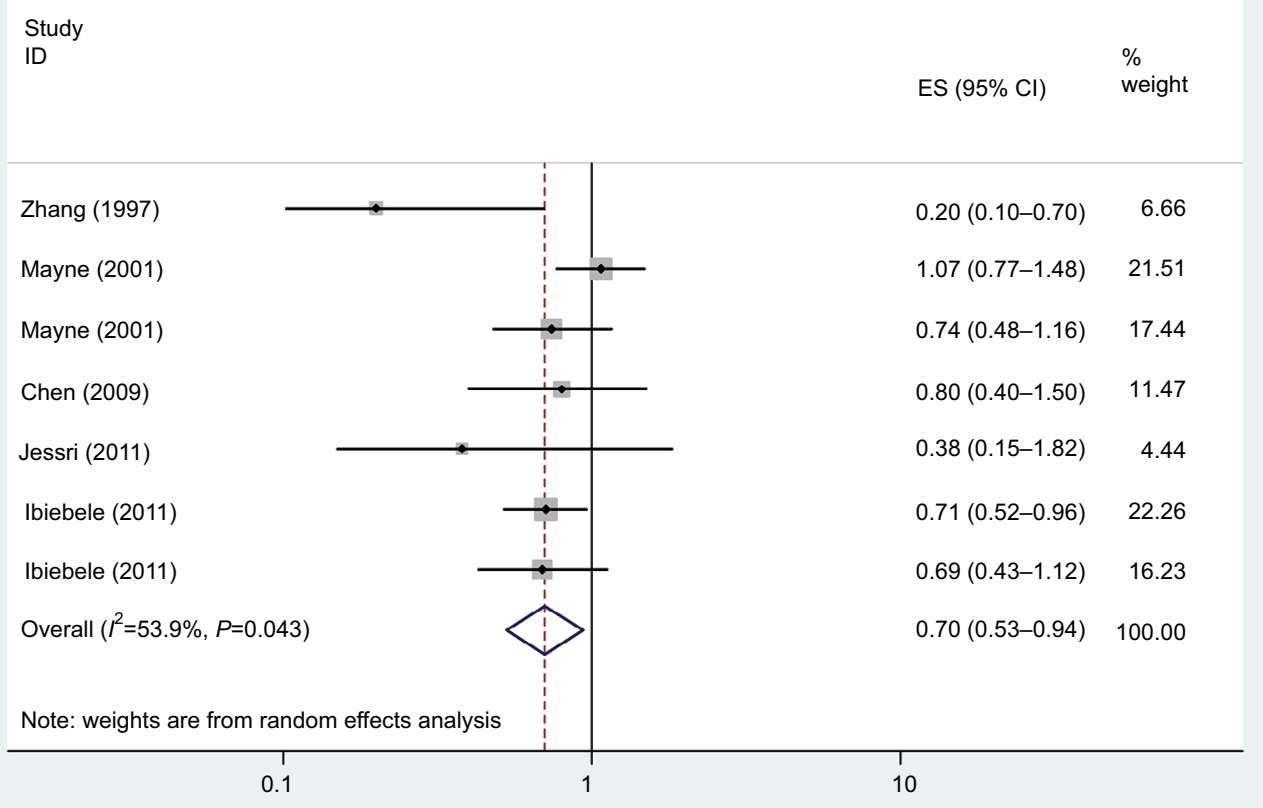

Figure 4 Forest plot between highest vs lowest categories of vitamin B3 intake and esophageal cancer risk.

Abbreviation: ES, .

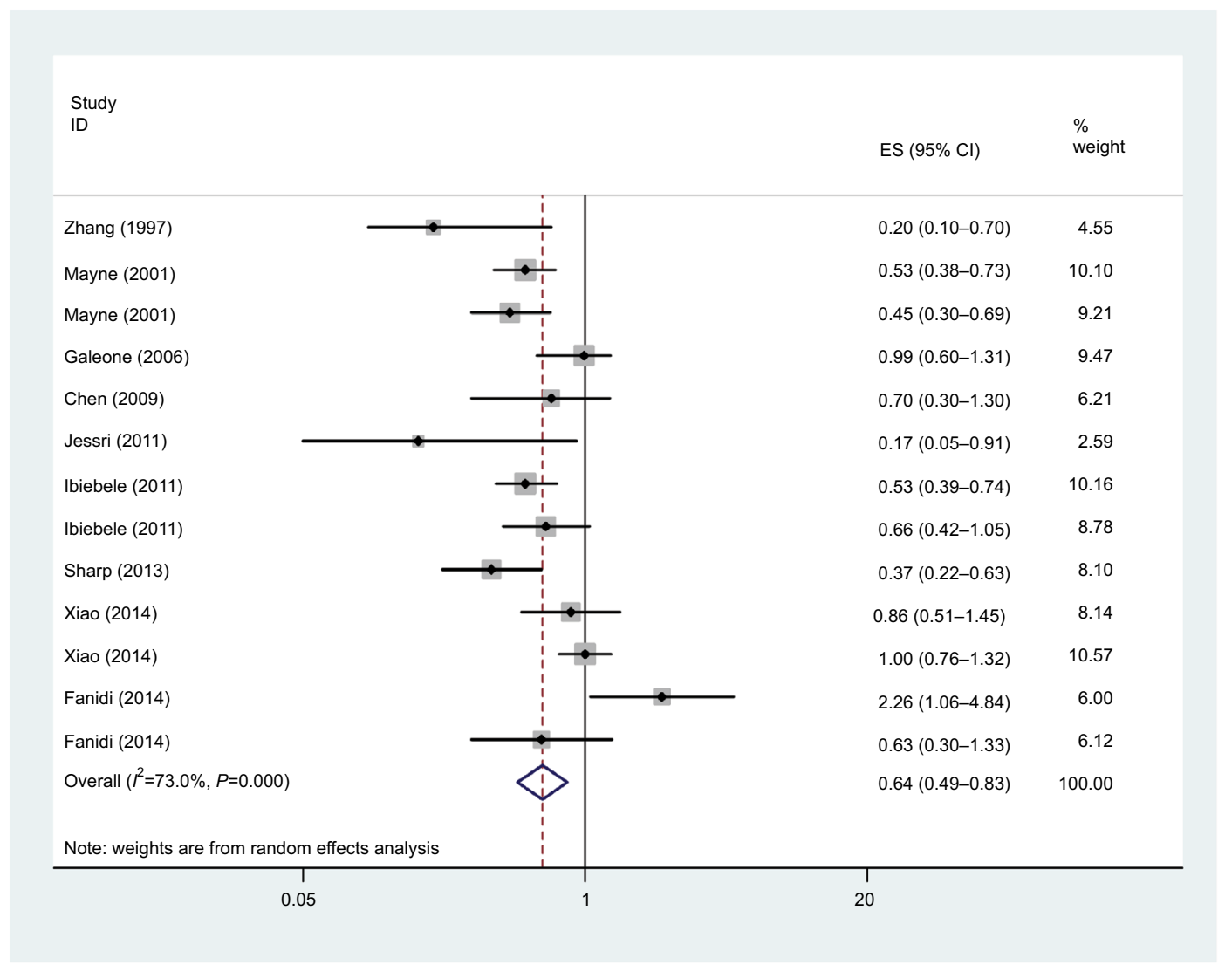

Figure 5 Forest plot between highest vs lowest categories of vitamin B6 intake and esophageal cancer risk. Abbreviation: ES, . 


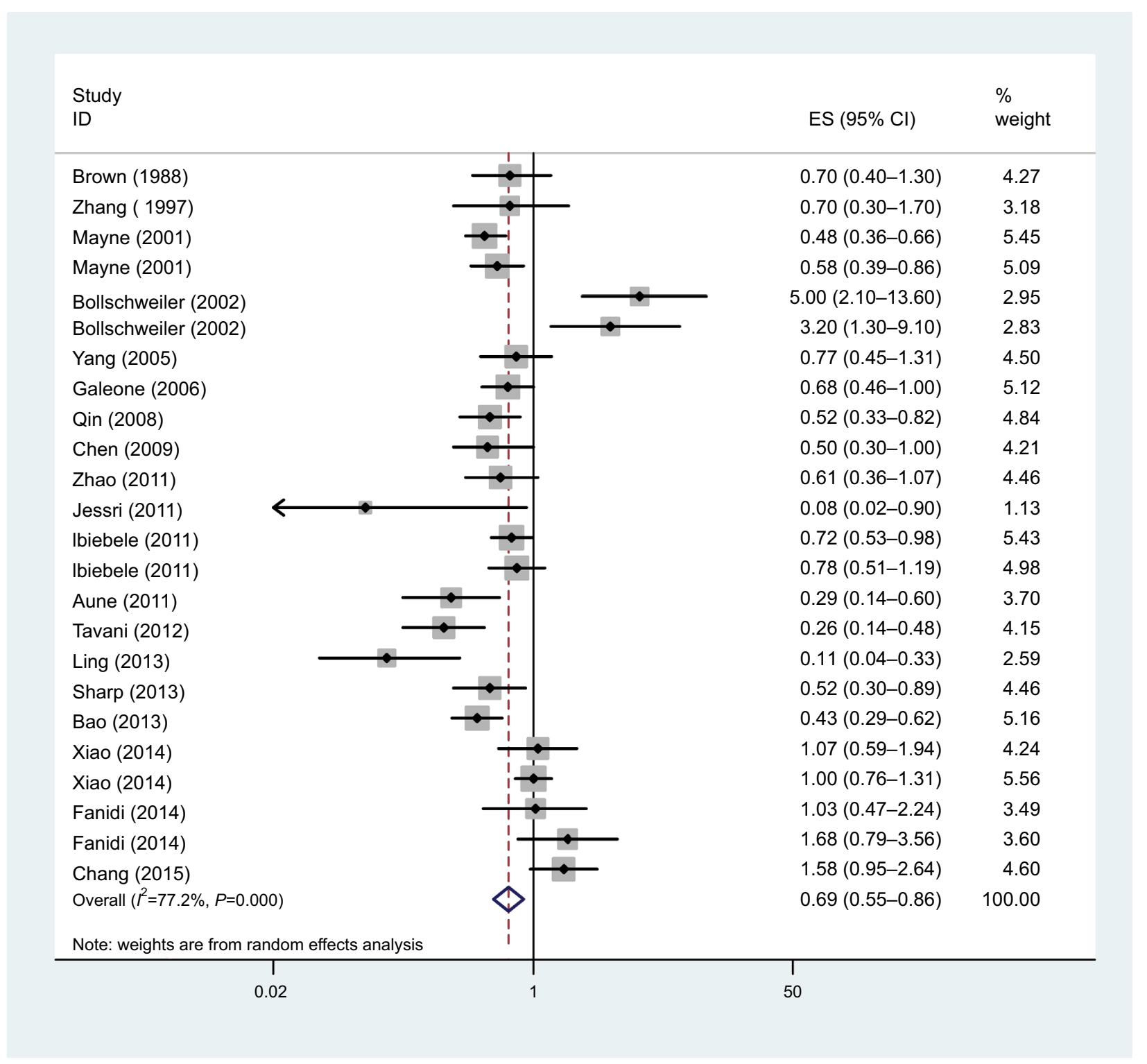

Figure 6 Forest plot between highest vs lowest categories of vitamin B9 intake and esophageal cancer risk. Abbreviation: ES, .

CI: $1.05-1.70)$. Heterogeneity was high $\left(I^{2}=73.6 \%, P=0.00\right)$, as shown in Figure 7. Using restricted cubic spline function, we found a potential non-linear dose-response association between dietary vitamin $\mathrm{B} 12$ intake and $\mathrm{EC}$ risk $\left(P_{\text {non-linearity }}=\right.$ 0.0001 ) (Figure 8 ). The non-linear curve showed that there was a dose-response association between vitamin B12 dose and decreased risk of EC approximately below $5.5 \mu \mathrm{g} /$ day, whereas the EC risk did not decrease further above $5.5 \mu \mathrm{g} /$ day.

\section{Publication bias}

Publication bias was evaluated by Egger' ${ }^{24}$ and Begg's tests. ${ }^{25}$ The results disclosed no evidence of publication bias for $\mathrm{EC}$ (Egger: $t=0.38, P=0.575$; Begg: $z=1.34 P=0.179$ ).

\section{Sensitivity analysis}

As a result, a sensitivity analysis of multivitamin B intake was conducted, and after each study was sequentially excluded from the pooled analysis, the conclusion was not affected by exclusion of any specific study.

\section{Discussion}

Epidemiological investigations have suggested that there are significant relationships between diet-associated factors and EC. B vitamins may be one factor. Because some B vitamins cannot be synthesized in the human body, they can only be obtained through dietary. Fruit and vegetables are important dietary sources of some B vitamins. The reason why vitamin 


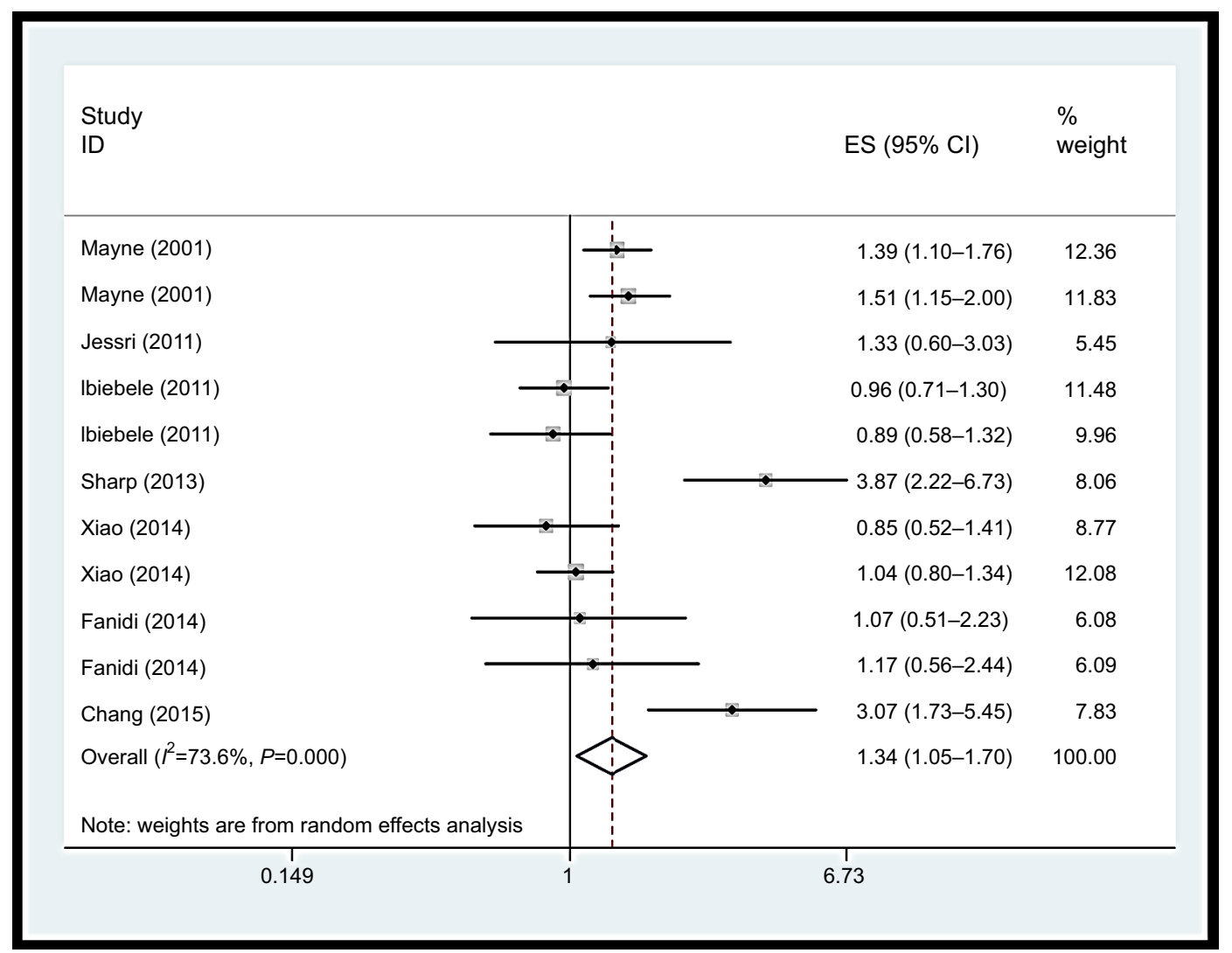

Figure 7 Forest plot between highest vs lowest categories of vitamin BI2 intake and esophageal cancer risk. Abbreviation: ES, .

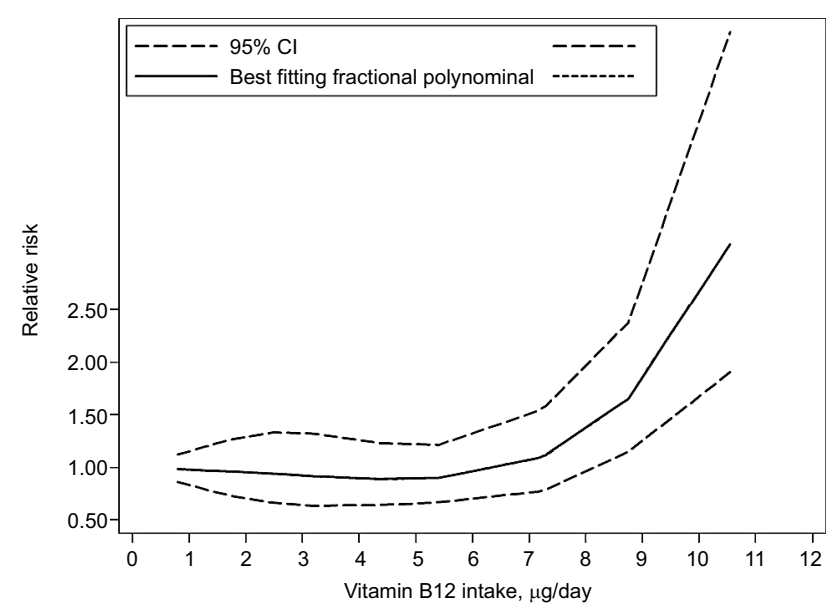

Figure 8 Non-linear dose-response analysis on vitamin BI2 intake and esophageal cancer risk.

$B$ affects the risk of cancer may be because it is essential for the biosynthesis of nucleotides, replication of DNA, supply of methyl groups, and the growth and repair of cells. ${ }^{43-46}$

In the present review, there was no epidemiologic research that assessed the association between total $\mathrm{B}$ vitamin consumption and EC risk among people. There were only studies which evaluated the relationship between several subclasses of B vitamins and EC risk. Thus, this study is the most comprehensive meta-analysis providing evidence to indicate these results. We found that total vitamin B intake was significantly associated with reduced $\mathrm{EC}$ risk. In addition, we evaluated the potential association of vitamin B subclasses and EC risk, respectively. In the subgroup analysis, we found that vitamin B1, B3, B6, and B9 may be protective factors, but vitamin B12, in contrast, was positively associated with risk of EC.

Previous studies have shown that consuming large quantities of vegetables, fruit, vitamins, and antioxidants can reduce the risk of EC..$^{47-49}$ One potential reason for vitamin B12 being different from other B vitamins may be because it is derived exclusively from foods of animal origin, and it is simply a marker for consumption of animal protein. In previous studies, the risk of adenocarcinomas of the esophagus was linked to high-fat diets ${ }^{50,51}$ because esophageal adenocarcinoma generally arises from Barrett's epithelium. ${ }^{52}$ Additionally, research has shown that diets low in animal protein and rich in fruit, vegetables, and fiber can reduce the risk of malignant transformation..$^{33,47}$ 
B-group vitamin supplementation may have antioxidant and anti-inflammatory effects. ${ }^{53,54}$ The biological mechanisms responsible for the protective effect of high-dosage vitamin $\mathrm{B}$ are unclear. One possible explanation is that $\mathrm{B}$ vitamins and additional nutrients sourced from fruit and vegetables are involved in the one-carbon metabolism. ${ }^{55-57}$ The metabolic pathway of one-carbon metabolism has been frequently implicated in carcinogenesis, because of its involvement in maintaining nucleotide biosynthesis and methylation reactions. Imbalances and deficiencies among crucial one-carbon metabolism nutrients may interfere with DNA replication, DNA repair, and regulation of gene expression, any of which could promote carcinogenesis. ${ }^{58,59}$ Like the vitamin B3, vitamin B6 and vitamin B9, they are indispensable in the biosynthesis of four bases of DNA (thymidine, guanine, adenine, and cytosine). Deficiency of one or more of the three vitamins required for DNA maintenance is known to cause abnormal pairing of the four bases, which can then result in mutations and the development of cancer. ${ }^{60}$ Intake of vitamin B6 was reported to increase immunoglobulin G and T4(helper) lymphocytes in humans. ${ }^{61}$ Folate deficiency was suggested to be related to increased carcinogenesis, an effect that may be mediated through participation in methyl metabolism. $^{62}$

\section{Limitations}

There were some limitations in our study that should be addressed. First, most studies included in our analysis were case-control studies, which may have caused recall bias, and could have caused potential heterogeneity, although the methodological quality of these observational studies was medium to high. More prospective cohort studies are needed to test this association. Second, it was a challenge to evaluate the quantity of vitamin B intake accurately because vitamin B can be sourced from various food types, and may be influenced by the type of cultivation, crop variety and location, as well as the specific morphological part of the plant eaten.

In conclusion, results from the present meta-analysis indicate that vitamin B intake is inversely associated with EC risk.

\section{Conclusion}

Our findings support that vitamin B may have an influence on carcinogenesis of the esophagus. Vitamin B1, B3, B6, and B9 showed a decreased risk of EC, vitamin B12 showed an increased risk of EC. (It is clear that scientists must apply the very best science in characterizing the safety of vitamin supplements.)

\section{Acknowledgment}

This work was funded by the MiaoPu Foundation of Henan Cancer Hospital.

\section{Disclosure}

The authors report no conflicts of interest in this work.

\section{References}

1. Ferlay J, Soerjomataram I, Dikshit R, et al. Cancer incidence and mortality worldwide: sources, methods and major patterns in GLOBOCAN 2012. Int J Cancer. 2015;136(5):E359-E386.

2. Di Pardo BJ, Bronson NW, Diggs BS, Thomas CR Jr, Hunter JG, Dolan JP. The Global Burden of Esophageal Cancer: A Disability-Adjusted Life-Year Approach. World J Surg. 2016;40(2): 395-401.

3. Boyle P, Levin B. World cancer report Lyon, France: IARC Press. 2008.

4. Muñoz N. Epidemiological aspects of oesophageal cancer. Endoscopy. 1993;25(9):609-612.

5. Wang JM, Xu B, Rao JY, Shen HB, Xue HC, Jiang QW. Diet habits, alcohol drinking, tobacco smoking, green tea drinking, and the risk of esophageal squamous cell carcinoma in the Chinese population. Eur $J$ Gastroenterol Hepatol. 2007;19(2):171-176.

6. Morita M, Kumashiro R, Kubo N, et al. Alcohol drinking, cigarette smoking, and the development of squamous cell carcinoma of the esophagus: epidemiology, clinical findings, and prevention. Int J Clin Oncol. 2010;15(2):126-134.

7. Falk GW. Risk factors for esophageal cancer development. Surg Oncol Clin NAm. 2009;18(3):469-485.

8. Galeone C, Pelucchi C, Levi F, et al. Folate intake and squamous-cell carcinoma of the oesophagus in Italian and Swiss men. Ann Oncol. 2006;17(3):521-525.

9. Aune D, Deneo-Pellegrini H, Ronco AL, et al. Dietary folate intake and the risk of 11 types of cancer: a case-control study in Uruguay. Ann Oncol. 2011;22(2):444-451.

10. World Cancer Research Fund/American Institute for Cancer Research. Food, nutrition, physical activity and the prevention of cancer; A global perspective. Washington, DC: AICR; 2007.

11. Sanjoaquin MA, Allen N, Couto E, Roddam AW, Key TJ. Folate intake and colorectal cancer risk: A meta-analytical approach. Int J Cancer. 2005;113(5):825-828.

12. Larsson SC, Giovannucci E, Wolk A. Folate intake, MTHFR polymorphisms, and risk of esophageal, gastric, and pancreatic cancer: a meta-analysis. Gastroenterology. 2006;131(4):1271-1283.

13. Chainani-Wu N. Diet and oral, pharyngeal, and esophageal cancer. Nutr Cancer. 2002;44(2):104-126.

14. Terry P, Lagergren J, Ye W, Nyrén O, Wolk A. Antioxidants and cancers of the esophagus and gastric cardia. Int J Cancer. 2000;87(5):750-754.

15. Tuyns AJ, Riboli E, Doornbos G, Péquignot G. Diet and esophageal cancer in Calvados (France). Nutr Cancer. 1987;9(2-3):81-92.

16. Decarli A, Liati P, Negri E, Franceschi S, La Vecchia C. Vitamin A and other dietary factors in the etiology of esophageal cancer. Nutr Cancer. 1987;10(1-2):29-37.

17. Nomura AM, Ziegler RG, Stemmermann GN, Chyou PH, Craft NE. Serum micronutrients and upper aerodigestive tract cancer. Cancer Epidemiol Biomarkers Prev. 1997;6(6):407-412.

18. Wells G, Shea B, O'Connell D, et al. The Newcastle-Ottawa Scale (NOS) for assessing the quality of nonrandomised studies in meta-analyses. Ottawa (ON): Ottawa Hospital Research Institute; 2009. Available from: http://www.ohri.ca/programs/. Accessed September 12, 2018.

19. Higgins JP, Green S. Cochrane Handbook for Systematic Reviews of Interventions, Version 5.1.0; 2011. Available from: https://training. cochrane.org/handbook. Accessed September 12, 2018. 
20. Chak E, Rutherford GW, Steinmaus C. The role of breast-feeding in the prevention of Helicobacter pylori infection: a systematic review. Clin Infect Dis. 2009;48(4):430-437.

21. Li Y, Yang H, Cao J. Association between Alcohol Consumption and Cancers in the Chinese Population-A Systematic Review and MetaAnalysis. PLoS One. 2011;6(4):e18776.

22. Deeks JJ, Higgins JPT, Altman DG. Analyzing data and undertaking metaanalyses, Ch. 9. In: Higgins J, Oxford Green S, editors. Cochrane Handbook for Systematic Reviews of Interventions 5.0.1. UK: The Cochrane Collaboration; 2008.

23. Higgins JPT, Thompson SG, Deeks JJ, Altman DG. Measuring inconsistency in meta-analyses. BMJ. 2003;327(7414):557-560.

24. Egger M, Smith GD, Schneider M, Minder C. Bias in meta-analysis detected by a simple, graphical test. BMJ. 1997;315(7109):629-634.

25. Begg CB, Mazumdar M. Operating characteristics of a rank correlation test for publication bias. Biometrics. 1994;50(4):1088-1101.

26. Bollschweiler E, Wolfgarten E, Nowroth T, Rosendahl U, Mönig S, Hölscher A. Vitamin intake and risk of subtypes of esophageal cancer in Germany. J Cancer Res Clin Oncol. 2002;128(10):575-580.

27. Huang GL, Wang SK, Su M, et al. Serum folate, MTHFR C677T Polymorphism and Esophageal Squamous Cell Carcinoma Risk. Biomed Environ Sci. 2013;26(12):1008-1012.

28. Sharp L, Carsin AE, Cantwell MM, Anderson LA, Murray LJ; FINBAR Study Group. Intakes of Dietary Folate and Other B Vitamins are Associated with Risks of Esophageal Adenocarcinoma, Barrett's Esophagus, and Reflux Esophagitis. J Nutr. 2013;143(12):1966-1973.

29. Zhao P, Lin F, Li Z, Lin B, Lin J, Luo R. Folate intake, methylenetetrahydrofolate reductase polymorphisms, and risk of esophageal cancer. Asian Pac J Cancer Prev. 2011;12(8):2019-2023.

30. Jessri M, Rashidkhani B, Hajizadeh B, Jessri M, Gotay C. Macronutrients, vitamins and minerals intake and risk of esophageal squamous cell carcinoma: a case-control study in Iran. Nutr J. 2011;20:10-137.

31. Chang S-C, Goldstein BY, Mu L, et al. Plasma folate, vitamin B12, and homocysteine and cancers of the esophagus, stomach, and liver in a Chinese population. Nutr Cancer. 2015;67(2):212-223.

32. Ibiebele TI, Hughes MC, Pandeya N, et al; Study of Digestive Health; Australian Cancer Study. High intake of folate from food sources is associated with reduced risk of esophageal cancer in an Australian population. J Nutr. 2011;141:274-283.

33. Mayne ST, Risch HA, Dubrow R, et al. Nutrient intake and risk of subtypes of esophageal and gastric cancer. Cancer Epidemiol Biomarkers Prev. 2001;10:1055-1062.

34. Bao L, Peng J, Huang G, et al. The study on the relationship between serum folic acid and vitamin B2 levels and esophageal cancer. Wei Sheng Yan Jiu. 2013;42(3):451-454. Chinese.

35. Fanidi A, Relton C, Ueland PM, et al. A prospective study of one-carbon metabolism biomarkers and cancer of the head and neck and esophagus. Int $J$ Cancer. 2015;136(4):915-927.

36. Tavani A, Malerba S, Pelucchi C, et al. Dietary folates and cancer risk in a network of case-control studies. Ann Oncol. 2012;23(10): 2737-2742.

37. Zhang ZF, Kurtz RC, Yu GP. Adenocarcinomas of the esophagus and gastric cardia: The role of diet. Nutr Cancer. 1997;27(3):298-309.

38. Qin JM, Wang XM, Chen B, et al. Study on the ingestion of folate and polymorphism of MTHFR C677T with esophageal cancer in Xinjiang Kazakh. Zhonghua Liu Xing Bing Xue Za Zhi. 2008;29(1):30-33. Chinese.

39. Brown LM, Blot WJ, Schuman SH, et al. Environmental factors and high risk of esophageal cancer among men in coastal South Carolina. J Natl Cancer Inst. 1988;80(20):1620-1625.

40. Chen H, Tucker KL, Graubard BI, et al. Nutrient Intakes and Adenocarcinoma of the Esophagus and Distal Stomach. Nutr Cancer. 2002;42(1):33-40.
41. Yang C-X, Matsuo K, Ito H, et al. Gene-environment interactions between alcohol drinking and the MTHFR C677T polymorphism impact on esophageal cancer risk: results of a case-control study in Japan. Carcinogenesis. 2005;26(7):1285-1290.

42. Xiao Q, Freedman ND, Ren J, Hollenbeck AR, Abnet CC, Park Y. Intakes of folate, methionine, vitamin B6 and vitamin B12 with risk of esophageal and gastric cancer in a large cohort study. Br J Cancer. 2014;110(5):1328-1333.

43. Smith AD, Kim YI, Refsum H. Is folic acid good for everyone? Am J Clin Nutr. 2008;87(3):517-533.

44. Liu L, Wylie RC, Andrews LG, Tollefsbol TO. Aging, cancer and nutrition: the DNA methylation connection. Mech Ageing Dev. 2003;124(10-12):989-998.

45. Ebbing $\mathrm{M}, \mathrm{B} ø$ naa $\mathrm{KH}$, Nygård $\mathrm{O}$, et al. Cancer incidence and mortality after treatment with folic acid and vitamin B12. JAMA. 2009;302(19):2119-2126.

46. Zhou YH, Tang JY, Wu M, et al. Effect of folic acid supplementation on cardiovascular outcomes: a systematic review and meta-analysis. PLoS One. 2011;6(9):e25142.

47. Navarro Silvera SA, Mayne ST, Gammon MD, et al. Diet and lifestyle factors and risk of subtypes of esophageal and gastric cancers: classification tree analysis. Ann Epidemiol. 2014;24(1):50-57.

48. Brown LM, Swanson CA, Gridley G, et al. Adenocarcinoma of the Esophagus: Role of Obesity and Diet. J Natl Cancer Inst. 1995;87(2):104-109.

49. Palli D, Bianchi S, Decarli A, et al. A case-control study of cancers of the gastric cardia in Italy. Br J Cancer. 1992;65(2):263-266.

50. Kabat GC, Ng SK, Wynder EL. Tobacco, alcohol intake, and diet in relation to adenocarcinoma of the esophagus and gastric cardia. Cancer Causes Control. 1993;4(2):123-132.

51. Tzonou A, Lipworth L, Garidou A, et al. Diet and risk of esophageal cancer by histologic type in a low-risk population. Int J Cancer. 1996;68(3): 300-304.

52. Kim R, Weissfeld JL, Reynolds JC, Kuller LH. Etiology of Barrett's metaplasia and esophageal adenocarcinoma. Cancer Epidemiol Biomark Prev. 1997;6(5):369-377.

53. Ullegaddi R, Powers HJ, Gariballa SE. B-group vitamin supplementation mitigates oxidative damage after acute ischaemic stroke. Clin $\mathrm{Sci}$ (Lond). 2004;107(5):477-484.

54. Ullegaddi R, Powers HJ, Gariballa SE. Antioxidant supplementation with or without B-group vitamins after acute ischemic stroke: a randomized controlled trial. JPEN J Parenter Enteral Nutr. 2006;30(2):108-114.

55. Olsen A, Halkjær J, van Gils $\mathrm{CH}$, et al. Dietary intake of the watersoluble vitamins B1, B2, B6, B12 and C in 10 countries in the European Prospective Investigation into Cancer and Nutrition. Eur J Clin Nutr. 2009;63(Suppl 4):S122-S149.

56. United States Department of Health and Human Services USDoA. Dietary Guidelines for Americans, U.S Dept. of Health and Human Services 2005. Washington DC: US Dept of Agriculture; 2005.

57. WCRF/AICR, Food, nutrition, physical activity and the prevention of cancer. A global perspective. USA: World Cancer Research Fund International; 2007.

58. Choi SW, Mason JB. Folate and carcinogenesis: an integrated scheme. J Nutr. 2000;130(2):129-132.

59. Kim Y. Folate and carcinogenesis: evidence, mechanisms, and implications. J Nutr Biochem. 1999;10(2):66-88.

60. Folkers K. Relevance of the biosynthesis of coenzyme Q10 and of the four bases of DNA as a rationale for the molecular causes of cancer and a therapy. Biochem Biophys Res Commun. 1996;224(2):358-361.

61. Folkers K, Morita M, McRee J Jr. The Activities of Coenzyme Q10 and Vitamin B6 for Immune Responses. Biochem Biophys Res Commun. 1993;193(1):88-92.

62. Rogers AE. Methyl donors in the diet and responses to chemical carcinogens. Am J Clin Nutr. 1995;61(3 Suppl):659S-665S. 


\section{Publish your work in this journal}

Cancer Management and Research is an international, peer-reviewed open access journal focusing on cancer research and the optimal use of preventative and integrated treatment interventions to achieve improved outcomes, enhanced survival and quality of life for the cancer patient. The manuscript management system is completely online and includes a very quick and fair peer-review system, which is all easy to use. Visit http://www.dovepress.com/testimonials.php to read real quotes from published authors.

Submit your manuscript here: https://www.dovepress.com/cancer-management-and-research-journal 